\title{
HIV-Associated Oral Kaposi Sarcoma: Case Report and Literature Review
}

Machado $\mathrm{MDC}^{* 1}$, Caixeirinho $\mathrm{P}^{1}$, Matos $\mathrm{I}^{2}$ and Marques $\mathrm{R}^{1}$

${ }^{1}$ Department of Stomatology, University Hospital Center of Lisbon Central, Lisbon, Portugal

${ }^{2}$ Department of Maxillofacial Surgery, University Hospital Center of Lisbon Central, Lisbon, Portugal

*Corresponding author: Machado MDC, Resident, Department of Stomatology, University Hospital Center of Lisbon Central, Lisbon, Portugal, Tel: + 351912374779, E-mail: ceu.machado.pt@gmail.com

Citation: Machado MDC, Caixeirinho P, Matos I, Marques R (2019) HIV-Associated Oral Kaposi Sarcoma: Case Report and Literature Review. J Case Rep Stud 7(2): 206. doi: 10.15744/2348-9820.7.206

Received Date: April 02, 2019 Accepted Date: April 25, 2019 Published Date: April 27, 2019

\begin{abstract}
Kaposi sarcoma (KS) is an angioproliferative polymorfic disorder that might be associated with human immunodeficiency virus (HIV) infection. In this clinical form, oral manifestation is particularly common and, sometimes, the first manifestation of the retroviral infection. In those cases, KS diagnosis is fundamental as the treatment is based in HIV infection control through an advisable multidisciplinary approach.
\end{abstract}

Keywords: Kaposi Sarcoma; Oral; Immunodeficiency; Highly Active Antiretroviral Therapy

\section{Introduction}

Kaposi Sarcoma (KS) is a systemic, angioproliferative disorder, caused by human herpes virus 8 (HHV-8) infection [1,2]. KS was first described by a Hungarian dermatologist named Moritz Kaposi in 1872 when this entity was identified as multi centric lesions in five patients, all of whom demised within 2 to 3 years $[3,4]$.

Subsequently, four major clinical forms have been identified with different patterns of evolution and prognosis. In Acquired Immunodeficiency Syndrome (AIDS) - associated KS, recognizing the oral manifestation of the disease is fundamental as the treatment is based on Human Immunodeficiency Virus (HIV) infection control. The authors present such a case, with oral KS as the first manifestation of the sarcomatous disease in a non-compliant HIV therapy patient.

\section{Case Report}

A 39 year-old Caucasian male patient, was sent to our Stomatology Department for augmented teeth mobility in the upper right quadrant. He had been diagnosed with HIV infection 4 years before and had past intensive care admission for crytococcal meningitis and pneumocystis pneumonia, but still refused antiretroviral therapy.

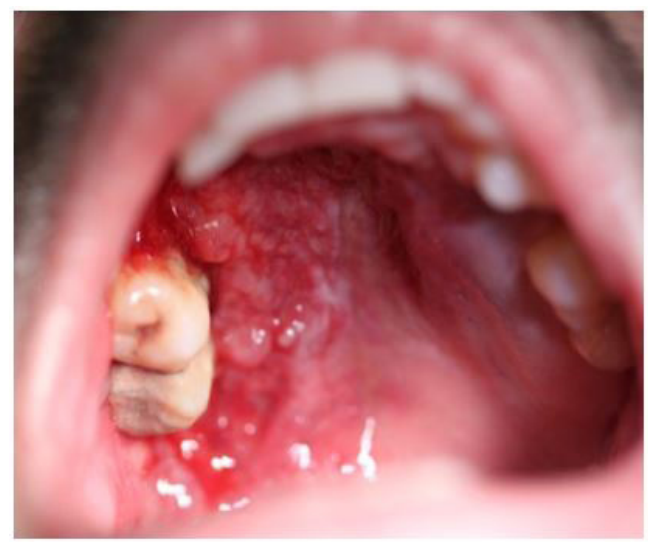

Figure 1: Intra-oral examination at Stomatology Department, Palatal Nodular and Eroded Mass

Detailed review of the medical history revealed that the patient previously reported a devalued small red spot in the upper right hard palate. However, two months later a biopsy thereof revealed inconclusive features. Five months later, the patient noticed augment teeth mobility and was sent to our department for evaluation. Intra-oral examination revealed a red to violaceous nodular nodular lesion that was poorly delimited (Figure 1). The lesion occupied the region of the gingival ridge and upper right palate. 
There was midline extension and the surface was eroded. On palpation, we verified a friable and easily hemorrhagic tumor. Significant augmented teeth mobility was perceived in the upper right quadrant as well. The patient denied oro-antral and oronasal communication symptoms and no other lesions were found, including skin lesions.

Incisional biopsies, panoramic X-ray (Figure 2) and maxillofacial computed tomography (CT) scan were performed (Figure 2a). Analytical work up showed HIV 1 load of 259692, 0 copies per milliliter and CD3+/CD4+ (Helper T Cells) of 0, 93\%.

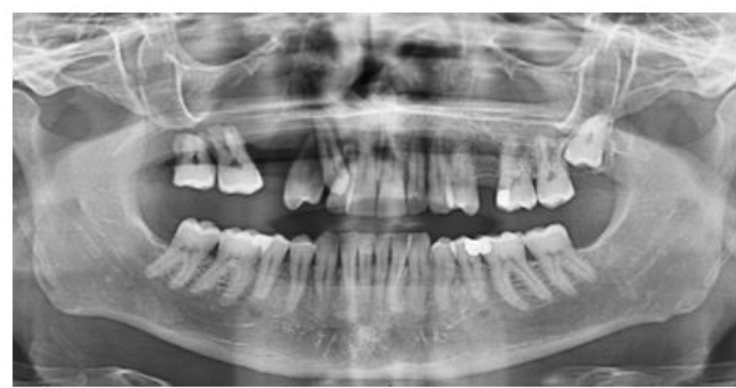

Figure 2: Panoramic X-ray, Bone Erosion on the Upper Right Quadrant

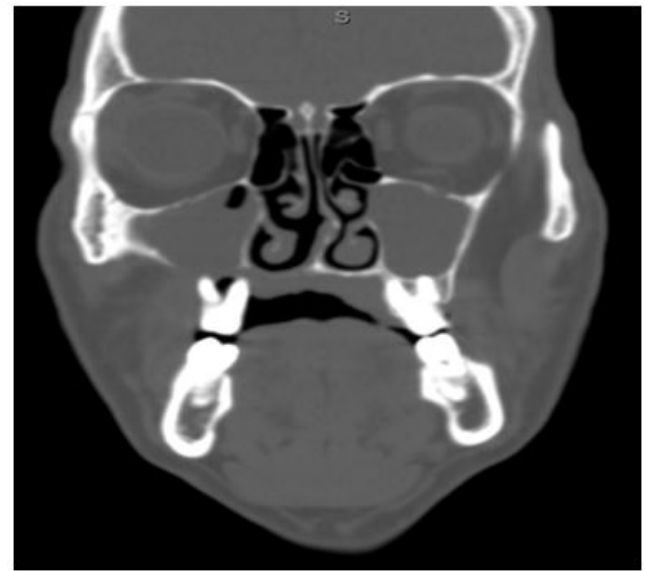

Figure 2A: Maxillofacial CT Scan, Extensive erosion of right maxillary sinus and nasal cavity floor, First right superior teeth with loss of bone support. Mucosa contour without augmented global thickness

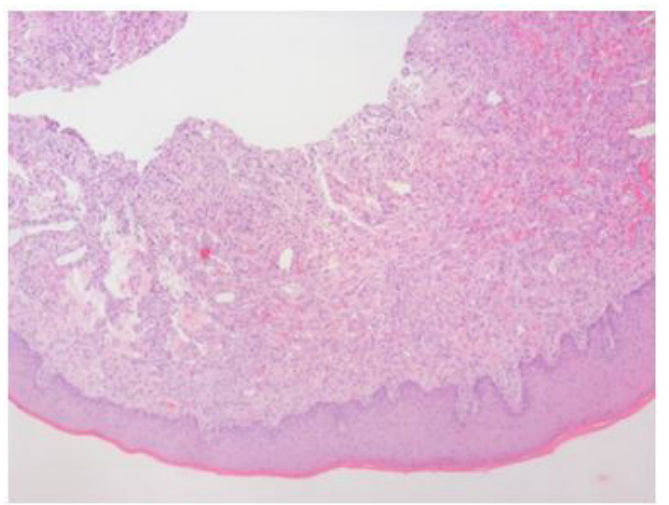

Figure 3: HE (x40 magnification), KS Comprising Slit-Like Vascular Spaces, Plump Spindle Cells and Extravagated Red Blood Cells

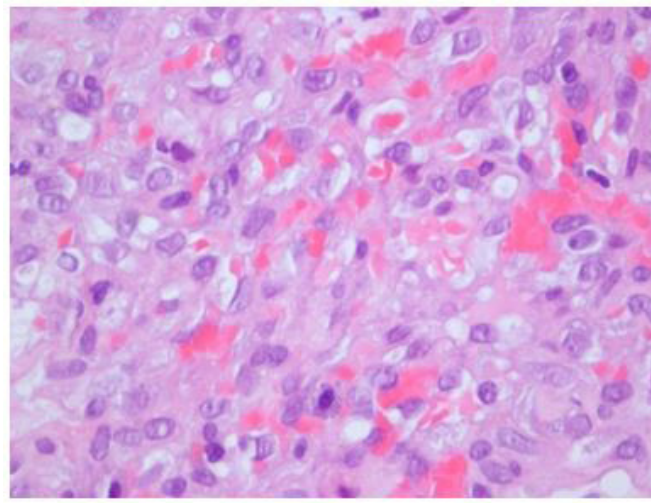

Figure 4: HE (x400 Magnification), Cellular Nuclear Pleomorphism 


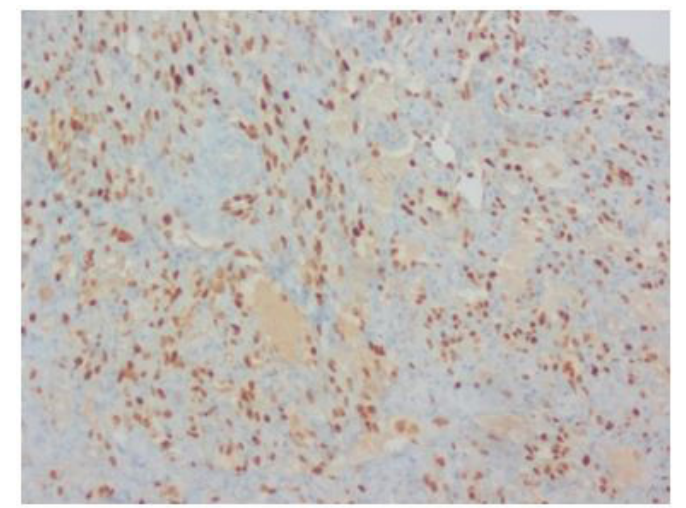

Figure 5: HHV-8 immunohistochemistry displays nuclear reactivity in the spindle cells of KS

Imaging studies showed palate and alveolar bone destruction in the right maxillary sinus floor and nasal cavity, supporting Kaposi sarcoma or Lymphoma diagnosis. Histopathological assessment of the tissue confirmed the presence of KS that was HHV-8 immunoreactive. CD 31 was also found positive and CD 20 was negative. The patient was transfered to the Infectology department for antiretroviral treatment institution and further studies. Following biopsies confirmed maxillary bone infiltration by sarcoma and visceral gastric and lung tumor involvement as well. Unfortunately, he was lost for follow up (Figure 3,4 and 5).

\section{Discussion}

Kaposi Sarcoma is a soft tissue malignancy that originates from an endothelial proliferation [2]. According to some authors, KS remains as a controversial issue; in some opinions, early $\mathrm{KS}$ is a polyclonal dearangement that might progress to a true malignancy [4,5].

All KS are caused by HHV-8 infection, a herpes virus, and by so capable to persist through the life of the host with long latency periods. Although, HHV-8 is always present in the tumor, infection with this virus isn't KS synonym and the pathogenesis is still not clear; in KS patients, HHV-8 infected endothelial precursor cells seem capable to combine them into new vasculature or to proliferate as KS [6].

HHV-8 transmission occurs mainly via saliva, but sexual, vertical and via blood products are also described. Breastfeeding doesn't appear to be such a source [4]. According to a study, immunodeficiency is a KS enabler but not a sine qua non condition, since the disease may occur in relatively preserved immune system patients [7].

KS is a systemic disease, with potential involvement of skin, mucosa, internal visceral organs and lymph nodes. The incidence has dropped in Europe, since the advent of highly active antiretroviral therapy (HAART), but it's still the most common cancer in HIV infected patients in Africa [8,9].

The diagnosis is confirmed by Anatomical Pathology. KS is typically characterized by slit-like vasculature, endothelial cell proliferation, extravagated red blood cells and hemosiderin pigment. A positive staining for HHV-8 facilitates the diagnosis [10].

Most authors consider four major clinical forms in KS. Sporadic or Classic Kaposi's sarcoma, is more common in man, especially those with Mediterranean or Jewish ancestry [11]. Older age, life in abundant blood sucking insects areas, past treatment with quinine, and farming cereals are possible risk factors [12,13]. Typically, it has an indolent course and a cutaneous lower limb predilection. Oral involvement is a rare manifestation. African Kaposi's sarcoma was endemic in Africa before AIDS. It's a more aggressive form of the disease, characterized by a more progressive evolution and lymphadenopathic involvement. Oral involvement is more common than in the classic form. HIV negative oral KS represent 5\% of African endemic KS [14].

Iatrogenic or transplant-associated KS, is a result of HHV-8 reactivation in most cases although seroconversion is a possibility $[15,16]$. The immunosuppressive drugs, particularly cyclosporine, play an important role, since it reactivates HHV-8 from latency to lytic replication in tissue culture. In this population, KS may occur months to years after organ transplantation but the risk seems to peak in the first 2 years after transplantation [17]. The evolution is quite variable ranging from chronic course to rapid progression but spontaneous regression, or after immunosuppressive withdraws, are also possible scenarios. In the oral cavity, the incidence is very low [4]. Epidemic or AIDS-related KS, originally an AIDS-defining illness, is still one of the most common malignancies in HIV positive patients, but its incidence has been declining since HAART advent [4]. It may occur at any stage of HIV infection but it is more prevalent at a lower CD4+ T-cell count [18]. Nevertheless, patients with oral HIV-KS have higher mortality than patients with only cutaneous HIV-KS [18]. It presents a more unpredictable evolution, usually aggressive, although the course and prognosis have also improved with HAART therapy. HAART therapy doesn't seems to affect HHV-8 replication but decreases HHV-8 viral load [4]. On the other hand, HIV infected patients may have a paradoxal KS worsening in context of the immune reconstitution inflammatory syndrome (IRIS), some with fatal outcome [1,19]. According to a study, at the time of oral HIV-KS diagnosis, there were significantly more patients with multiple oral HIV-KS lesions than patients with single oral HIVKS lesion [18]. The same study observed other simultaneous oral mucosa changes, such as pseudomembranous candidiasis, hairy leukoplakia, and necrotizing gingivitis in descending order. 
Gross features in oral Kaposi sarcoma may be quite variable. In the oral cavity, the aspect can range from patch, plaque or nodular. The colour can vary from red to violaceous or even brown according to hemorrhagic and hemossiderin deposition in stroma tissue and increased melanocytes stimulation [7]. Palate is the most common spot involved, followed by gingiva and tongue, either as localized or multifocal lesions. Other sites include buccal mucosa, major salivary glands and jaw bones [10]. There has been reported three cases of KS presenting as diffuse gingival enlargement [2]. The authors stated a unique growth pattern for KS, since it extends beyond mucogingival junction to the alveolar mucosa in a fast growing way [2].

The treatment of HIV-associated KS is best achieved within a multidisciplinary approach. The infectiologist and maxillofacial surgeon, among others, are important resources for clinical discussion and treatment. Management may depend upon a variety of factors including results of staging and comorbilities. Local measures such as intralesional vinblastine or systemic administration of cytotoxic chemotherapy for disseminated disease are all possibilities [20].

\section{Conclusion}

Oral involvement in Kaposi sarcoma is a possibility in all clinical forms, but most commonly seen in AIDS associated KS. Meanwhile, since HAART advent, it became less frequent. This case, illustrates the destructive capacity of KS in an immunosuppressed patient. Mouth location, provides an easy biopsy approach to determine the diagnosis. The stomatologist, among other oral healthcare professionals, might be the first to detect the lesion, to confirm the diagnosis and to guide the patient to a better outcome.

\section{References}

1. Ramírez-Amador V, Anaya-Saavedra G, Martínez-Mata G (2010) Kaposi’s sarcoma of the head and neck: A review. Oral Oncol 46: 135-45.

2. Stern JK, Stern I, De Rossi SS, Zemse SM, Abdelsayed RA (2016) Kaposi sarcoma presenting as "diffuse gingival enlargement": Report of three cases. HIV AIDS Rev 15: 80-7.

3. Kaposi M (1872) Idiopathisches multiples Pigmentsarkom der Haut. Arch Dermatol. Syph 4: 265-73.

4. Schwartz RA, Micali G, Nasca MR, Scuderi L (2008) Kaposi sarcoma: a continuing conundrum. J Am Acad Dermatol 59: 179-206.

5. Duprez R, Lacoste V, Brière J, Couppié P, Frances C, et al. (2007) Evidence for a multiclonalorigin of multicentric advanced lesions of Kaposi sarcoma. J Natl Cancer Inst 99: 1086-94.

6. Radkov SA, Kellam P, Boshoff C (2000) The latent nuclear antigen of Kaposi sarcoma-associated herpesvirus targets the retinoblastoma-E2F pathway and with the oncogene Hras ransforms primary rat cells. Nat Med 6: 1121-7.

7. Hoffmann C, Sabranski M, Esser S (2017) HIV-Associated Kaposi’s Sarcoma. Oncol Res Treat 40: 94-8.

8. Fitzmaurice C, Dicker D, Pain A, Hamavid H, Moradi-Lakeh M, et al. (2015) The Global Burden of Cancer 2013. JAMA Oncol 1: 505-27.

9. Parkin DM, Bray F, Ferlay J, Jemal A (2014) Cancer in Africa 2012. Cancer Epidemiol Biomarkers Prev 23: 953-66.

10. Agaimy A, Mueller SK, Harrer T, Bauer S, Thompson LDR (2018) Head and Neck Kaposi Sarcoma: Clinicopathological Analysis of 11 Cases. Head Neck Pathol 12: 511-6.

11. Laor Y, Schwartz RA (1979) Epidemiologic aspects of American Kaposi’s sarcoma. J Surg Oncol 12: 299-303.

12. Ruocco V, Schwartz RA, Ruocco E (2002) Lymphedema: an immunologically vulnerable site for development of neoplasms. J Am Acad Dermatol 47: 124-7.

13. Cottoni F, Masala MV, Budroni M, Rosella M, Satta R, et al. (1997) The role of occupation and a past history of malaria in the etiology of classic Kaposi's sarcoma: a case control study in north east Sardinia. Br J Cancer 76: 1518-20.

14. Thariat J, Kirova Y, Sio T, Choussy O, Vees H, et al. (2012) Mucosal Kaposi sarcoma, a Rare Cancer Network study. Rare Tumors 4: 156-61.

15. Lebbé C, Legendre C, Francès C (2008) Kaposi sarcoma in transplantation. Transplant Rev (Orlando) 22: 252-61.

16. Parravicini C, Olsen SJ, Capra M, Poli F, Sirchia G, et al. (1997) Risk of Kaposi's sarcoma- associated herpes virus transmission from donor allografts among Italian posttransplant Kaposi's sarcoma patients. Blood 90: 2826-9.

17. Tessari G, Naldi L, Boschiero L, Cordiano C, Piaserico S, et al. (2006) Incidence and clinical predictors of Kaposi's sarcoma among 1721 Italian solid organ transplant recipientes: a multicentre study. Eur J Dermatol 16: 553-7.

18. Khammissa RA, Pantanowitz L, Feller L (2012) Oral HIV-Associated Kaposi Sarcoma: A Clinical Study from the Ga-Rankuwa Area, South Africa. AIDS Res Treat 2012: 1-9.

19. Fatahzadeh M, Schwartz RA (2013) Oral Kaposi's sarcoma: a review and update. Int J Dermatol 52: 666-72.

20. Carvalho L, Lucena L, Honorato M, Andrade G, Freitas R (2016) Mucocutaneous Kaposiss sarcoma in an HIV-positive patient: diagnosis and treatment. J Bras Patol Med Lab 52: 194-7. 


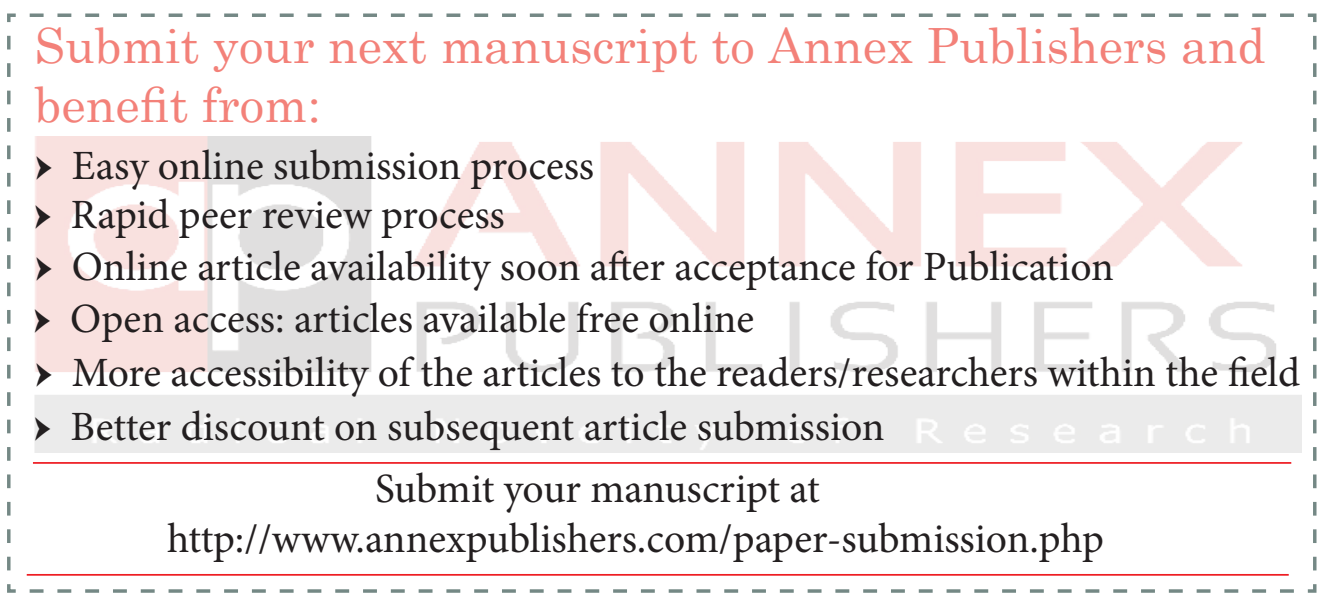

